\title{
Impacto de la educación digital en salud durante la pandemia COVID-19
}

\section{Impact of digital education on health during the pandemic COVID-19}

\author{
Pamela Rocio Picoy-Romero ${ }^{1,2, a}$ \\ ${ }^{1}$ Instituto de Investigación de Ciencias Biomédicas, Universidad Ricardo Palma. Lima, Perú. \\ ${ }^{2}$ Facultad de Medicina Humana, Universidad Ricardo Palma. Lima, Perú. \\ ${ }^{a}$ Estudiante de medicina humana, ORCID: https://orcid.org/0000-0001-7405-1618
}

Correspondencia:

Pamela Rocio Picoy Romero

pamepicoy94@gmail.com

Recibido: 25 de octubre 2020

Aprobado: 15 de diciembre 2020

Publicación en línea: 30 de diciembre

2020

Conflictos de interés: La autora declara

no tener conflictos de interés.

Fuente de financiamiento:

Autofinanciado

Citar como: Picoy-Romero P. Impacto de la educación digital en salud durante la pandemia COVID-19. An Fac med. 2020;81(3):368-9. DOI: https://doi.org/10.15381/anales. v81i3.19047.
An Fac med. 2020;81(3):368-9. / DOI: https://doi.org/10.15381/anales.v81i3.19047

\section{Sr. Editor,}

En tiempos de COVID-19, la situación de las prácticas clínicas ha cambiado de tal manera que se han visto en la obligación de eliminar el contacto directo con los pacientes y cambiar el aprendizaje presencial. Se ha demostrado que el aprendizaje en línea ayuda a fomentar el autoaprendizaje y tiene tanto éxito como la didáctica tradicional ${ }^{(1)}$.

La telesalud se ha convertido en un factor importante en la prestación de servicios. Dado su uso, la participación de los estudiantes de medicina parece no solo muy beneficioso para los propios estudiantes, sino también para los pacientes que necesitan atención. Los estudiantes pueden ser invitados a la sala virtual para participar en la toma de antecedentes, observar el examen físico virtual y ser parte de la toma de decisiones, el asesoramiento al paciente y la familia, y la planificación de implementaciones ${ }^{(1)}$.

Las interacciones del aprendizaje en línea y las intervenciones de telesalud se complementarían en un nuevo modelo de aprendizaje, el cual suscita la participación del paciente y ayuda al aprendizaje del estudiante sea más real. También está afectado las interacciones de aprendizaje basadas en problemas, disecciones de anatomía en persona, aprendizaje en grupo sesiones, exámenes locales y requisitos de licencia ${ }^{(1)}$.

Puede ser que las reuniones médicas futuras adopten un enfoque híbrido de combinar lo digital con la experiencia cara a cara ${ }^{(2)}$. Es posible que se necesite orientación regulatoria para desarrollar aplicaciones de telesalud seguras y amigables para el paciente (2). Se debe observar todos los factores predominantes en una buena educación virtual en salud, dando énfasis en la transparencia y comunicación para promover un aprendizaje óptimo, así como extender el uso de herramientas en línea.

En un estudio que evalúa el "estudio clínico estructurado objetivo", se identificaron y exploraron tres áreas de necesidad de desarrollo de competencias: competencia técnica; recopilación de información virtual, incluidos antecedentes, recopilación de información colateral y examen físico; y habilidades de comunicación interpersonal, tanto verbal como no verbal ${ }^{(3)}$.

Aún existen limitaciones sobre cómo adecuar tanto las aptitudes básicas para las habilidades de comunicación no verbal y sus herramientas estandarizadas para evaluarlas. Un análisis a profundidad reharía ciertos parámetros de las enseñanzas virtualizadas. La literatura actual en telemedicina tiene contribuciones significativas desde el campo de la dermatología, particularmente con el uso de tecnología store-and-forward o asincrónica ${ }^{(4)}$. 
Para los aprendices quirúrgicos en Italia, los residentes de urología han adaptado y utilizado tecnologías de aprendizaje inteligente que incluyen: una biblioteca de videos pregrabados con clases impartidas, consejos y trucos de expertos, seminarios web en vivo con comentarios de expertos, y discusión de casos clínicos, clubes de revistas de redes sociales y rondas virtuales ${ }^{(5)}$. Para los aprendices de cirugía que requieran revalidación antes de septiembre de 2020, se han otorgado extensiones. Como opción alternativa, se esta considerando el financiamiento para el aprendizaje en línea y a distancia durante COVID-19 ${ }^{(5)}$.

La telemedicina se convertirá en una herramienta relevante para adoptar nuevos sistemas de atención y educación médica, obligándonos a analizar las limitaciones y eficacias en su funcionamiento, para así crear nuevas formas de interacción con los estudiantes y residentes de medicina.

\section{REFERENCIAS BIBLIOGRÁFICAS}

1. Theoret C, Ming X. Our education, our concerns: The impact on medical student education of COVID-19. Med Educ. 2020;54(7): 591-592. DOI: 10.1111/medu.14181
2. Shah S, Diwan S, Kohan L, Rosenblum D, Gharibo C, Soin A, et al. The Technological Impact of COVID-19 on the Future of Education and Health Care Delivery. Pain Physician. 2020;23(4S):S367-S380.

3. Lawrence K, Hanley K, Adams J, Sartori DJ, Greene R, Zabar S. Building Telemedicine Capacity for Trainees During the Novel Coronavirus Outbreak: a Case Study and Lessons Learned. J Gen Intern Med. 2020;35(9):2675-2679. DOI: 10.1007/ s11606-020-05979-9

4. Shah P, Gutierrez D, Zampella J. Teaching Lessons from the Coronavirus Disease 2019 (COVID-19) Pandemic in Telemedicine. J Drugs Dermatol. 2020;19(6):677.

5. Al-Jabir A, Kerwan A, Nicola M, Alsafi Z, Khan M, Sohrabi C, et al. Impact of the Coronavirus (COVID-19) pandemic on surgical practice - Part 1. Int J Surg. 2020;79:168-179. DOI: 10.1016/j.jjsu.2020.05.022 